\section{Subdural Hematoma}

Elliot J. Roth

Department of Physical Medicine and

Rehabilitation, Northwestern University, Feinberg School of Medicine, Chicago, IL, USA

\section{Synonyms}

SDH

\section{Definition}

A subdural hematoma ( $\mathrm{SDH}$ ) is a bleeding inside the skull but outside of the brain, located on the surface of the brain, usually resulting from severe head trauma.

\section{Current Knowledge}

SDH occurs when there is tearing of the tiny bridging veins that connect the dura covering and the surface of the brain. In acute $\mathrm{SDH}$, the collection fills up with blood rapidly, compressing brain tissue, and resulting in brain damage and obvious symptoms. Chronic SDH tends to be more subtle, occurring after minor head trauma, with no or minimal symptoms, going unnoticed for days to weeks. Occasionally, SDH occurs spontaneously without obvious cause. SDH occurs with greater frequency in older adults than in younger persons because of the frequency of falls and the altered brain anatomy that places their bridging veins at greater risk of tearing. Anticoagulant use and alcohol abuse also increase the risk. Symptoms include lethargy or confusion, balance or gait difficulty, nausea or headache, seizure or visual disturbances, and weakness or sensory loss. Diagnosis is made on neuroimaging. Treatment involves immediate craniotomy and evacuation of the hematoma as promptly as possible. Outcome depends on the location and size of the hematoma and how quickly treatment is instituted. Long-term complications include seizures, cognitive dysfunction, hemiparesis, sensory loss, and other neurological deficits.

\section{Cross-References}

- Intracranial Hemorrhage

\section{References and Reading}

Maurice-Williams, R. S. (1999). Chronic subdural hematoma: An everyday problem for the neurosurgeon. British Journal of Neurosurgery, 13, 547-549.

Weigel, R., Schmiedek, P., \& Krauss, J. K. (2003). Outcome of contemporary surgery for chronic subdural haematoma: Evidence based review. Journal of Neurology, Neurosurgery \& Psychiatry, 74, 937-943. 